\section{Fatores sócio-culturais e éticos relacionados com os processos de diagnóstico da esquistossomíase mansônica em área de baixa endemicidade}

\author{
Socio-cultural and ethical factors involved \\ in the diagnosis of schistosomiasis mansoni \\ in an area of low endemicity
}

\author{
Margareth Maria Lessa Gonçalves 1 \\ Magali Muniz Gonçalves Barreto ${ }^{1}$ \\ Arnaldo Maldonado Jr. 1 \\ Vanessa Regal Maione 1 \\ Luís Rey 1 \\ Marisa da Silveira Soares 1
}

\footnotetext{
1 Instituto Oswaldo Cruz, Fundação Oswaldo Cruz, Rio de Janeiro, Brasil.

Correspondência M. M. L. Gonçalves Laboratório de Avaliação e Promoção de Saúde Ambiental, Instituto Oswaldo Cruz, Fundação Oswaldo Cruz. Av. Brasil 4365, Rio de Janeiro, $R J$ 21045-900, Brasil. margaremaria@yahoo.com.br
}

\begin{abstract}
Five annual parasitological surveys and one serological survey, respectively based on the Kato-Katz and free sedimentation methods and the Western blot technique, were conducted in Sumidouro, Rio de Janeiro, Brazil, an endemic county for schistosomiasis. Possible influences of the use of these methodologies on social, cultural, and ethical aspects of the study population were also evaluated. Having the opportunity to choose the different techniques was a conclusive issue influencing participation by the population. Prevalence rates of positive results for stool tests were: $11.6 \%$ (1995); 8.8\% (1996); 12.2\% (1998); 5.9\% (1999); and 3.2\% (2000). In the period during which the serological survey was performed, the use of laboratory testing in association with analysis of clinical data and available data on transmission and treatment generated a diagnostic procedure termed "coproseroepidemiology". This methodology contributed to significant improvements in the accuracy of measurement of local schistosomiasis prevalence, indicating that epidemiological surveillance could help prevent the recurrence of high prevalence rates. The fact that Biomphalaria glabrata was replaced by Melanoides tuberculata in the main transmission focus contributed to a significant decrease in infection rates.
\end{abstract}

Schistosomiasis Mansoni; Diagnosis; Endemic Diseases

\section{Introdução}

Desde o final dos anos 50 a população de um município brasileiro de pequeno porte (Sumidouro, Rio de Janeiro, Brasil) tem sido submetida a sucessivos "Programas de Controle da Esquistossomíase Mansônica”, sempre orientados verticalmente por órgãos federais de controle de endemias. As principais medidas adotadas foram a quimioterapia e a aplicação de moluscicidas nos focos de transmissão do Schistosoma mansoni. Houve também, ações modestas em educação e uma tentativa, precocemente interrompida, de implantação de fossas sépticas. Nos últimos anos, os cortes orçamentários sofridos pelos órgãos executores dessas campanhas de controle, reduziram as ações à quimioterapia baseada em diagnósticos bienais realizados em uma fração da área endêmica, com método reconhecidamente insuficiente para detectar positivos em situações de baixa endemicidade - Kato-Katz com uma amostra de fezes $1,2,3,4$.

Em 1994, a situação da esquistossomíase em Sumidouro era típica de baixa endemicidade 5,6, mas com elevada transmissibilidade focal, ocorrência de alguns casos de hepatoesplenomegalia e de hepatomegalia e queixas diversas entre os indivíduos que apresentavam a forma intestinal da doença. Em 1995, nossos resultados baseados em um inquérito parasitológico preliminar sugeriram um quadro local 
da esquistossomíase mais severo do que se supunha até então. Os percentuais de positividade chegaram a $10 \%$, o que já seria motivo suficiente para se investir em métodos mais sensíveis de diagnóstico.

Outro motivo relevante para aperfeiçoar o diagnóstico em Sumidouro seria a necessidade de avaliar correlações entre as taxas de infecção em humanos e no reservatório silvestre do $S$. mansoni, o roedor Nectomys squamipes, abundante na região $7,8,9$. As técnicas que melhor atenderiam a essas demandas por sensibilidade, teoricamente seriam as baseadas em análises sorológicas ou na análise de múltiplas amostras de fezes 4 . Entretanto, o elevado percentual de recusas à participação por parte de alguns moradores no inquérito coprológico realizado em 1995, foi superior ao desejado em situações de baixa endemicidade. No contexto local, para que o diagnóstico fosse aperfeiçoado, seria necessário se compreender os fatores subjacentes às recusas à participação e, para isso, seria importante que se desse atenção não apenas às técnicas, mas a todo o processo, levando em consideração, por exemplo, a interveniência de fatores sociais, culturais e éticos. O presente trabalho avalia o diagnóstico da esquistossomíase em áreas de baixa endemicidade, como processo dependente desses múltiplos fatores.

\section{Metodologia}

O trabalho descreve e analisa, em bases interdisciplinares, observações realizadas pela equipe entre 1995 e 2000 em quatro localidades próximas à sede do Município de Sumidouro. O molusco hospedeiro intermediário do S. mansoni nessas localidades foi Biomphalaria glabrata.

Pedreira, localidade periurbana próxima à zona rural, com abastecimento de água tratada e atividade econômica de seus moradores exercida principalmente na zona urbana. Porteira Verde, Pamparrão e Santa Cecília são as localidades rurais. Praticamente todos os seus residentes trabalham em minifúndios distribuídos pelas três localidades. Pela diversidade eco-epidemiológica, tanto Porteira Verde como Pamparrão foram divididas em duas áreas: Alta menor densidade demográfica, coleções hídricas menos poluídas por esgotos, ausência de criadouros de moluscos; Baixa - maior densidade demográfica, coleções hídricas mais poluídas por esgotos e presença de moluscos.
Segundo a população local, a nossa equipe foi a primeira a penetrar em uma área de difícil acesso (Porteira Verde Alta), onde residiam sete famílias, para realizar algum tipo de trabalho assistencial. A população residente nas quatro localidades era de cerca de seiscentas pessoas.

A população e a equipe tiveram variadas oportunidades de contato: houve visitas anuais às famílias para cadastramento, preenchimento de questionários (para obtenção de dados sócio-econômicos) e realização de entrevistas (sobre percepções da população a respeito da esquistossomíase e sobre coleções hídricas freqüentadas). Anualmente era feito o diagnóstico coprológico e a quimioterapia da esquistossomíase e demais parasitoses, sendo a última etapa de tratamento realizada em 2001. Em 1996 e 1999, foram realizadas atividades educativas (feiras de saúde e ambiente, debates com a comunidade, dinâmica com livro infantil) abertas à participação de toda a população e que eram pautadas pelo diálogo entre todos os participantes (pesquisadores, donas-de-casa, estudantes locais, lavradores, comerciantes, aposentados etc.). Todas essas oportunidades de contato foram aproveitadas pela equipe para estabelecer, eticamente, laços de confiança com a população, que resultaram em cerca de noventa dias de informações registradas por observação participante 10 .

A experiência mais objetiva de escolha de método diagnóstico pela população ocorreu em 1996, quando a infecção humana pelo $S$. mansoni foi avaliada por coprologia e por sorologia.

Nesse ano, antes da etapa de diagnóstico, a equipe visitou cada residência informando à população sobre três fatos: em primeiro lugar, que o "exame do sangue" serviria apenas para diagnosticar a esquistossomíase, não se prestando, portanto, a outros diagnósticos (glicemia, anemia etc.); em segundo, sobre a complementaridade dos métodos, ou seja, a sua insuficiência como método isolado; em terceiro lugar, sobre a possibilidade de optar por ambos, por um apenas ou por nenhum deles; finalmente, sobre o fato de que, no exame de fezes seriam adotados dois métodos que, juntos, detectariam grande número de enfermidades, inclusive "giárdia" e "ameba", reconhecidos pela própria população como comuns na região.

Após esses esclarecimentos, as seguintes perguntas, formuladas de acordo com o vocabulário local, foram dirigidas a 178 dos 345 indivíduos acima dos dez anos de idade: "Para saber se tem o 'verme do caramujo', você prefere 
fazer exame de fezes, exame de sangue ou os dois tipos de exames? Por quê?".

A coprologia baseou-se nas técnicas de Kato-Katz e de sedimentação espontânea 11,12, com a colheita de até três amostras de fezes, em dias diferentes, sendo preparadas duas lâminas por amostra. As amostras de soro foram submetidas à reação imunoenzimática western blot para determinação de anticorpos IgG para esquistossomíase, empregando-se como antígeno extrato solúvel de $S$. mansoni 13 .

Assumindo-se como premissas: (a) a complementaridade dos métodos laboratoriais adotados neste trabalho para diagnóstico da esquistossomíase, constatada empiricamente em estudos prévios na mesma área geográfica; (b) a probabilidade elevada de infecções passadas serem detectadas pela sorologia, por se tratar de área endêmica 4 e (c) com a probabilidade reduzida de detecção de casos pela coprologia em áreas de baixa endemicidade 1,3 , criou-se o diagnóstico coprosoroepidemiológico, resultante da união dos resultados obtidos por coprologia e por sorologia confirmada tanto pela coprologia como por critérios clínicos e epidemiológicos. Os critérios clínicos de positividade foram as formas hepatointestinal e hepatoesplênica. O critério epidemiológico baseouse tanto no contato com focos de transmissão como no histórico de tratamentos anteriores 14 . Os focos de transmissão eram locais onde constatava-se a existência de moluscos infectados pelo $S$. mansoni. O tratamento para a esquistossomíase ocorrido em um período inferior ou igual a 12 meses foi um critério de exclusão.

\section{Análises de dados}

As preferências das comunidades foram investigadas pelas intenções manifestas nas respostas à pergunta formulada antes da fase de diagnóstico e pelo método a que cada indivíduo, efetivamente, se submeteu. As comparações quantitativas entre intenções ou entre freqüências de participações e de não participações foram feitas pelas provas de Fisher ou de qui-quadrado $\left(\chi^{2}\right)$. Possíveis associações entre ter sorologia positiva para o $S$. mansoni e estar infectado por outro helminto ou algum protozoário foram investigadas pelos testes de independência ( $\chi^{2}$ ou Fisher).

Este trabalho obteve parecer favorável do Comitê de Ética em Pesquisa do Instituto Oswaldo Cruz, Fundação Oswaldo Cruz.

\section{Resultados}

\section{Participação}

A avaliação realizada em 1996, ofereceu a possibilidade de escolha durante o inquérito parasitológico e revelou que 176 (99\%) dentre os 178 entrevistados tinham a intenção de participar do diagnóstico por pelo menos um dos métodos. Noventa e nove (56\%) dos 178 entrevistados tinham a intenção de participar por ambos os métodos oferecidos. Quarenta e um (23\%) deles pretendiam participar apenas pela coprologia e 37 (21\%) apenas pela sorologia.

Os que declararam querer se submeter a ambos os diagnósticos, alegaram fazê-lo por ser "melhor", "mais apurado", e porque "qualquer um é bom”. Trinta e seis (90\%) dentre os 41 entrevistados que optaram pela coprologia declararam não gostar de colher sangue. Diziam eles: "Não gosto de tirar sangue", "Deus me perdoe, exame de sangue só quando tem filho”, “Deus me livre", "De sangue, de jeito nenhum”.

Dentre os 37 que optaram pela sorologia, 16 (43\%) declararam considerá-la mais eficiente do que o exame de fezes ("De fezes já fiz uma porção de vezes”, “O de fezes sempre dá negativo”, “ $O$ de fezes, a maioria já fez e sempre deu negativo”). Seis indivíduos consideraram a sorologia um método mais prático e conveniente ("De sangue não dá amolação", "Não consigo fazer de fezes porque sou ressecada”, "É bom mudar”, “De fezes não consigo porque faço no trabalho”).

Do total de 178 entrevistados, $28 \%$ mostraram não ter compreendido o objetivo do exame, apesar dos esclarecimentos prestados pelo entrevistador ("Nunca fiz exame de sangue", "Não faço há muito tempo", "Acho que tenho sangue fraco", "Tenho alguma coisa no sangue", "Tenho anemia", "Sinto coisa mordendo que parece caramujo”).

A participação efetiva, na fase de diagnóstico, por pelo menos um dos métodos foi de $86 \%$ dentre os 518 moradores cadastrados no ano de 1996. Em cada sexo, nos grupos entre 11 e 40 anos de idade e em Porteira Verde Baixa, a participação por um método implicou a participação também pelo outro. Nos demais grupos as opções foram independentes. Crianças e indivíduos com mais de quarenta anos preferiram a coprologia (Tabela 1 ).

Das 405 pessoas que participaram da coprologia, $56 \%$ forneceram as três amostras fecais, $28 \%$ entregaram duas e $16 \%$ entregaram apenas uma. A coprologia identificou 13 positivos dentre os que não participaram da sorologia.

A sorologia evidenciou 15 positivos dentre os que não participaram da coprologia, 12 dos 
Participação da população de localidades de Sumidouro, Rio de Janeiro, Brasil, nos inquéritos coprológico e sorológico da esquistossomíase (1996): percentuais e resultados dos testes de Fisher ou de qui-quadrado para avaliação da independência na escolha pelo método diagnóstico.

\begin{tabular}{|c|c|c|c|c|c|c|c|c|c|}
\hline & \multicolumn{2}{|c|}{ Coprologia } & \multicolumn{2}{|c|}{ Sorologia } & \multicolumn{2}{|c|}{ Ambos } & \multicolumn{2}{|c|}{ Nenhum } & \multirow[t]{2}{*}{$\mathbf{P} \chi^{2}$} \\
\hline & $\mathrm{n}$ & $\%$ & $\mathrm{n}$ & $\%$ & $\mathrm{n}$ & $\%$ & $\mathrm{n}$ & $\%$ & \\
\hline \multicolumn{10}{|l|}{ População/sexo } \\
\hline População & 173 & 33 & 40 & 8 & 232 & 45 & 73 & 14 & 0,000 \\
\hline Masculino & 97 & 35 & 19 & 7 & 123 & 44 & 42 & 15 & 0,000 \\
\hline Feminino & 76 & 32 & 21 & 9 & 109 & 46 & 31 & 13 & 0,018 \\
\hline \multicolumn{10}{|c|}{ Grupos etários (em anos) } \\
\hline $1-10$ & 59 & 54 & 4 & 4 & 37 & 34 & 9 & 8 & 0,763 \\
\hline $11-20$ & 45 & 34 & 11 & 8 & 50 & 38 & 25 & 19 & 0,024 \\
\hline $21-30$ & 19 & 23 & 8 & 10 & 41 & 49 & 16 & 19 & 0,003 \\
\hline $31-40$ & 16 & 9 & 3 & 2 & 47 & 25 & 8 & 4 & 0,004 \\
\hline$>40$ & 34 & 28 & 14 & 12 & 57 & 48 & 15 & 13 & 0,170 \\
\hline \multicolumn{10}{|l|}{ Localidades } \\
\hline Porteira Verde Alta & 46 & 32 & 11 & 8 & 80 & 56 & 6 & 4 & 0,922 \\
\hline Porteira Verde Baixa & 44 & 34 & 7 & 5 & 66 & 50 & 14 & 11 & 0,0241 \\
\hline Pamparrão Alto & 3 & 14 & 1 & 5 & 16 & 76 & 1 & 5 & 0,352 \\
\hline Pamparrão Baixo & 19 & 43 & 1 & 2 & 13 & 30 & 11 & 25 & 0,068 \\
\hline Pedreira & 50 & 34 & 16 & 11 & 51 & 35 & 30 & 20 & 0,076 \\
\hline Santa Cecília & 11 & 34 & 4 & 13 & 6 & 19 & 11 & 34 & 0,712 \\
\hline
\end{tabular}

Nota: valores em negrito simbolizam associação estatística positiva.

quais confirmados por critérios epidemiológicos e clínicos. Destes, cinco eram possíveis contaminadores de criadouros do molusco B. glabrata por meio de esgotos domésticos. A sorologia evidenciou, também, que sete indivíduos que recusaram-se a participar da coprologia eram negativos, apesar do contato peridomiciliar com focos de transmissão.

\section{Prevalências}

A Tabela 2 mostra as prevalências de infecção pelo $S$. mansoni, baseadas em inquéritos parasitológicos (coprologia) realizados no período de cinco anos.

Na Tabela 3 encontram-se as prevalências referentes ao ano de 1996, estimadas pelos diferentes métodos diagnósticos. A coprologia mostrou proporções elevadas de positivos em grupos específicos: sexo masculino, grupo entre 11 e 20 anos de idade, trabalhadores em agropecuária, moradores em área contendo focos de transmissão e moradores de Porteira Verde Baixa e Pamparrão Alto e Baixo. Pela coprosoroepidemiologia as prevalências foram ainda mais elevadas, chegando a ultrapassar os $60 \%$. Dentre os 445 participantes com amostras de sangue e/ou de fezes, foram identificados 130 positivos para o $S$. mansoni. Separadamente cada método mostrou prevalências de $8 \%$ na coprologia, $40 \%$ na sorologia e $34 \%$ na coprosoroepidemiologia.

As prevalências da infecção considerandose apenas os 232 indivíduos que se submeteram a todos os métodos de diagnóstico foram de $15 \%$ pela coprologia, $40 \%$ pela sorologia e de $35 \%$ pela coprosoroepidemiologia.

O percentual de positividade obtido pela coprologia com três amostras (10\%) foi o dobro do obtido com apenas uma amostra (5\%).

As prevalências encontradas para outros parasitos intestinais foram: $13 \%$ para ancilostomídeos; $5 \%$ para Trichuris trichiura; $5 \%$ para Ascaris lumbricoides; 20\% para Entamoeba coli; $10 \%$ para Giardia lamblia. Houve independência entre ser positivo na sorologia para $S$. mansoni e estar infectado por outro helminto ou protozoário. Os resultados dos testes estatísticos que averiguaram a ocorrência dessa possível associação foram: $\mathrm{p} \chi^{2}=0,323$ para ancilostomídeos; pFisher $=0,358$ para $T$. trichiura; $\mathrm{p} \chi^{2}=0,192$ para A. lumbricoides; $\mathrm{p} \chi^{2}=0,742$ para $E$. coli e p $\chi^{2}=0,356$ para G. lamblia.

Todos os indivíduos com resultado parasitológico positivo, passaram por exame clínico 
Prevalências da infecção pelo Schistosoma mansoni obtidas por meio dos inquéritos parasitológicos realizados no período de 1995 a 2000, em localidades de Sumidouro, Rio de Janeiro, Brasil.

\begin{tabular}{|c|c|c|c|c|c|c|c|c|c|c|}
\hline & \multicolumn{10}{|c|}{ Coprologia } \\
\hline & \multicolumn{2}{|c|}{1995} & \multicolumn{2}{|c|}{1996} & \multicolumn{2}{|c|}{1998} & \multicolumn{2}{|c|}{1999} & \multicolumn{2}{|c|}{2000} \\
\hline & $n$ & $\%$ & $\mathrm{n}$ & $\%$ & $\mathrm{n}$ & $\%$ & $n$ & $\%$ & $\mathrm{n}$ & $\%$ \\
\hline \multicolumn{11}{|c|}{ População/sexo } \\
\hline Masculino & 250 & 14,4 & 222 & 11,3 & 218 & 14,2 & 260 & 6,2 & 228 & 4,8 \\
\hline Feminino & 199 & 8,0 & 186 & 5,9 & 192 & 9,9 & 212 & 5,7 & 210 & 1,4 \\
\hline Total & 449 & 11,6 & 405 & 8,8 & 410 & 12,2 & 472 & 5,9 & 438 & 3,2 \\
\hline
\end{tabular}

Nota: valores em negrito destacam as prevalências para a população.

Tabela 3

Prevalências da infecção pelo Schistosoma mansoni, obtidas no ano de 1996 pelos métodos diferentes de diagnóstico realizados em localidades de Sumidouro, Rio de Janeiro, Brasil.

\begin{tabular}{|c|c|c|c|c|c|c|}
\hline & \multicolumn{2}{|c|}{ Coprologia } & \multicolumn{2}{|c|}{ Sorologia } & \multicolumn{2}{|c|}{$\begin{array}{l}\text { Coprosoroe- } \\
\text { pidemiologia }\end{array}$} \\
\hline & $\mathrm{n}$ & $\%$ & $n$ & $\%$ & $\mathrm{n}$ & $\%$ \\
\hline \multicolumn{7}{|l|}{ População/sexo } \\
\hline População & 405 & 9 & 272 & 40 & 232 & 35 \\
\hline Masculino & 220 & 11 & 142 & 43 & 123 & 40 \\
\hline Feminino & 185 & 6 & 130 & 36 & 109 & 29 \\
\hline \multicolumn{7}{|c|}{ Grupos etários (em anos) } \\
\hline $1-10$ & 96 & 3 & 41 & 15 & 37 & 14 \\
\hline $11-20$ & 95 & 18 & 61 & 36 & 50 & 34 \\
\hline $21-30$ & 60 & 8 & 49 & 47 & 41 & 44 \\
\hline $31-40$ & 63 & 6 & 50 & 56 & 47 & 45 \\
\hline$>40$ & 91 & 8 & 71 & 41 & 57 & 35 \\
\hline \multicolumn{7}{|l|}{ Localidades } \\
\hline Porteira Verde Alta & 126 & 4 & 91 & 21 & 80 & 11 \\
\hline Porteira Verde Baixa & 110 & 16 & 73 & 56 & 66 & 67 \\
\hline Pamparrão Alto & 19 & 21 & 17 & 35 & 16 & 31 \\
\hline Pamparrão Baixo & 32 & 28 & 14 & 57 & 13 & 69 \\
\hline Pedreira & 101 & 1 & 67 & 49 & 51 & 28 \\
\hline Santa Cecília & 17 & 0 & 10 & 10 & 6 & 0 \\
\hline \multicolumn{7}{|l|}{ Ocupação } \\
\hline Agropecuária & 152 & 15 & 111 & 47 & 97 & 44 \\
\hline Outra & 212 & 6 & 150 & 35 & 125 & 28 \\
\hline \multicolumn{7}{|l|}{ Foco de transmissão } \\
\hline Presente & 140 & 19 & 86 & 57 & 78 & 68 \\
\hline Ausente & 265 & 4 & 186 & 32 & 154 & 18 \\
\hline
\end{tabular}

Nota: valores em negrito destacam as prevalências. e receberam o tratamento correspondente aos helmintos e protozoários intestinais. No caso da esquistossomíase, aqueles positivos pela coproscopia e/ou soroepidemiologia foram examinados e, conforme orientação médica, 96 deles receberam praziquantel $(40 \mathrm{mg} / \mathrm{kg}$ de peso do paciente).

\section{Outras observações relevantes}

Numa área outrora negligenciada pelas campanhas de controle, Porteira Verde Alta, a participação com as três amostras de fezes em 1995 foi de $100 \%$. Em 1996, a participação por pelo menos um dos métodos foi de $96 \%$, a maior dentre todas as seis áreas.

Apenas duas pessoas externaram preocupação quanto à qualidade do material usado na sorologia ("A agulha é descartável?", "Se for com seringa boa...”).

\section{Discussão}

Os resultados confirmaram que o diagnóstico da esquistossomíase em comunidades de áreas de baixa endemicidade depende tanto da eficiência das técnicas como da decisão individual de participar, sendo, portanto, inútil encará-lo como evento eminentemente tecnológico. É necessário percebê-lo como um processo dependente da interação de vários fatores (bioquímicos, culturais, éticos etc.). Os resultados sugerem que o diagnóstico seja, também, uma condição em que os indivíduos e grupos podem sentir-se expostos de várias maneiras. Assim sendo, a decisão de participar ou não dependeria da disposição de expor-se.

O principal questionamento em relação ao emprego da coprologia no caso em questão advém do fato de que a intensidade de infecção 
pelo S. mansoni era baixa (< 100 ovos/g de fezes), o que favorece a subestimação do número de infectados. Isto é importante porque, ainda que as infecções leves não diagnosticadas não sejam responsáveis pelo aparecimento de patologia grave, podem ser responsáveis pela persistência da transmissão. Outra importante consideração referente aos falsos negativos pelo exame de fezes, devido à infecção leve, é que esses indivíduos podem figurar como "novos casos" em inquéritos posteriores, gerando confusão na compreensão sobre reinfecção e aquisição de resistência 1 .

Apesar da limitação, a coprologia mostrouse imprescindível por: (a) mostrar, de forma inquestionável, que alguns dos que se recusaram a colher sangue eram positivos e tinham condições potencialmente favoráveis a contaminar focos de moluscos com ovos do S. manso$n i$; (b) ser um método prático e de baixo custo; (c) evidenciar o parasito diretamente; (d) permitir a contagem de ovos, que é indicativo da intensidade de infecção e base de cálculo para o Índice Potencial de Contaminação Ambiental 15; (e) oferecer a possibilidade de comparação com resultados de outros trabalhos.

O aumento de amostras de fezes examinadas dobrou a probabilidade de encontro dos infectados, demonstrando ser indispensável empreender esforços para o fornecimento das três amostras. Além disso, o exame da amostra fecal permite que numa mesma leitura ao microscópio sejam detectados diferentes parasitos intestinais, sem que haja qualquer encarecimento e, por outro lado, realize-se um controle mais abrangente, concedendo mais de uma forma de retorno à população.

É certo que ocorreram avanços no imunodiagnóstico da esquistossomíase, contudo, sempre limitados em decorrência do resultado rápido e barato fornecido pela coproscopia nas campanhas de controle de helmintíases, principalmente nos países em desenvolvimento, como o Brasil, onde os recursos financeiros destinados à pesquisa têm sido escassos. Somente na última década, consolidou-se a idéia de trabalhar-se com o imunodiagnóstico, ao ponto de intensificarem-se os investimentos em ensaios sorológicos padronizados que pudessem ser usados no campo ou na rotina de laboratórios, para identificação da esquistossomíase. A sorologia pode ser extremamente útil em situações como a de Sumidouro, pois, ao contrário da coprologia, tem muito menos probabilidade de fornecer falsos negativos, cuja presença frustraria qualquer programa de controle. $\mathrm{O}$ fato de pessoas escolherem a sorologia como diagnóstico foi importante por dois motivos: (a) evidenciou quais dos não participantes da coprologia seriam possíveis contaminadores dos criadouros de B. glabrata com ovos do $S$. mansoni e (b) mostrou, provavelmente com grande margem de acerto, os negativos residentes em área de risco.

Mas, a maior contribuição para a compreensão da situação epidemiológica foi dada pela coprosoroepidemiologia. Dada a complexidade da situação e as dificuldades de diagnóstico, a coprosoroepidemiologia certamente permitiu uma aproximação em relação à verdadeira prevalência local. Esse método composto, valendo-se da complementaridade entre coprologia, sorologia, epidemiologia e clínica, por um lado diminui a possibilidade de que se deixem positivos sem tratamento e por outro, de que se façam tratamentos desnecessários, que sempre foram repudiados 16 .

Os percentuais de positividade obtidos durante os cinco anos em que realizamos a coprologia, demonstram que o processo diagnóstico mais abrangente, que resultou numa maior cobertura quimioterápica em 1996, não impediu que houvesse um aumento da prevalência em 1998, fato compatível com a extrema precariedade sanitária da região. Por outro lado, a partir de 1999 as prevalências entraram em declínio, possivelmente não apenas devido à quimioterapia, mas pela redução da população de B. glabrata e do potencial de transmissão da doença motivados pelo estabelecimento de moluscos Melanoides tuberculata no principal sítio de transmissão 17,18,19,20 .

A população foi receptiva à participação no diagnóstico, ainda que nem todos por ambos os métodos. É presumível que a proposta de métodos novos (sorologia e coprologia com maior número de amostras) tenha estimulado a participação da comunidade. Por meio dos questionários, percebeu-se que alguns acreditavam que essa proposta efetivamente conduziria a diagnósticos da esquistossomíase mais acurados, enquanto outros imaginavam, equivocadamente, que ela solucionaria quaisquer problemas de saúde.

Contudo, convém discutir as causas da não participação, visto que, em alguns grupos, ela foi acentuada, o que é indesejável em área de baixa endemicidade, nas quais a detecção de positivos entre os participantes já é, por si só, um problema. Aparentemente, a disposição dos indivíduos em participar sofreu a influência de constrangimentos relacionados à colheita das fezes e à exposição destas à equipe de diagnóstico. Um sintoma desse constrangimento era o fato de que, de modo geral, a população ignorava o saco plástico fornecido pela equipe 
e embrulhava os frascos coletores em várias camadas de papel e, sobre elas, sacos plásticos grandes. Por isso eram consumidos cerca de noventa minutos para desempacotar as amostras recolhidas em um dia. Dois rapazes que sempre participaram do diagnóstico e, freqüentemente, estavam infectados por S. mansoni deixaram de fazê-lo após participarem de inúmeras conversações informais com membros da equipe.

É plausível supor, também, que houvesse receio de que o resultado fosse divulgado, pois Sumidouro é uma cidade pequena e, nas localidades onde o trabalho foi realizado, todos se conhecem. Uma questão a ser considerada é a dificuldade de garantir que portadores assintomáticos de ovos de S. mansoni, freqüentes em áreas de baixa endemicidade, como Sumidouro, submetam-se a métodos de diagnóstico e a tratamentos que considerem desagradáveis.

Outra questão relacionada ao comportamento humano e que pode ter interferido nos resultados é a participação fraudulenta, com fezes de animais ou de outras pessoas. Um indivíduo confessou que um membro de sua família havia colocado fezes de porco no frasco. Após ser visitada por quatro dias consecutivos para recolhimento dos frascos de sua família, durante os quais sempre se desculpava por "não colaborar", uma dona-de-casa entregou todas as quatro amostras. Duas delas eram idênticas entre si e tinham aspecto e odor inusitados para fezes humanas. Neste aspecto, o exame de sangue apresenta mais uma vantagem: a colheita de material depende da presença do próprio indivíduo a ser examinado (devidamente identificado), assegurando a origem da amostra.

Todas essas causas de recusa e de fraude precisam ser neutralizadas para que se conte com o maior número possível de participantes no diagnóstico. Os constrangimentos podem ser neutralizados, ainda que não se consiga eliminá-los, pela percepção dos benefícios individuais e coletivos que o diagnóstico pode propiciar e pela confiança dos indivíduos em quem o realiza. Para estimular essa percepção, a informação deve atingi-los de modo a sensibilizá-los, resguardados os limites da ética. É necessário que eles percebam, sem serem cons- trangidos, que seus interesses individuais, apesar de legítimos, contrapõem-se a outros interesses igualmente legítimos: os do restante da comunidade, já que recusas ao diagnóstico e ao tratamento propiciam a disseminação local da esquistossomíase.

Nesses casos, a educação apoiada pela antropologia se apresenta como instrumento ideal não só para informar sobre a necessidade de participar adequadamente em todas as formas de diagnóstico oferecidas, mas também para permitir e fomentar o diálogo. Com base na antropologia, a educação se aperfeiçoa como "via de mão dupla, de entendimento global das situações através do exercício da troca virtual de papéis e não como via de mão única para imposição de informação que visem apenas facilitar diagnósticos ou outras intervenções posteriores" 21 (p. 107). Por outro lado, novamente observando-se a ética, devem ser informados de que sua privacidade será garantida pela não divulgação dos resultados a pessoas externas à equipe, da mesma forma que será assegurado o seu direito a não participar do diagnóstico.

Apesar de ambos os métodos serem questionáveis sob vários aspectos, o fato de muitos indivíduos terem optado por apenas um deles não deve ser encarado como frustrante, mas sim como real. Por esse prisma, pode-se considerar que a abordagem adotada atendeu plenamente ao desejado para a realidade local.

É importante destacar que foram muitos os motivos alegados para a escolha do método diagnóstico. Por respeitar as diferenças individuais, a possibilidade de opção foi um fator decisivo para que a comunidade aceitasse participar. Os métodos são complementares tanto para diagnóstico individual, pois aumentam a probabilidade de um portador de ovos ser identificado como tal, como também em relação à evidenciação dos casos na população. Assim, não há sentido em comparar coprologia e sorologia, mas sim usar uma como auxiliar da outra, sempre que possível.

Outro ponto evidenciado neste trabalho foi a discordância entre as intenções manifestas e as opções efetivas, que expôs o equívoco de se investigar comportamentos influenciáveis por medos e tabus a partir apenas de questionários. 


\section{Resumo}

Foram realizados em Sumidouro, Rio de Janeiro, Brasil, município endêmico para a esquistossomíase, cinco inquéritos parasitológicos anuais e um sorológico, baseados nas técnicas de Kato-Katz, sedimentação espontânea e western blot. Avaliou-se ainda a interveniência de fatores sociais, culturais e éticos na aplicação de tais métodos junto à comunidade. A possibilidade de opção foi decisiva para a participação das pessoas. As prevalências pela coproscopia foram: 11,6\% (1995); 8,8\% (1996); 12,2\% (1998); 5,9\% (1999); 3,2\% (2000). No ano de realização da sorologia, os resultados dos exames laboratoriais associados às informações sobre a transmissão, o acompanhamento clínico dos infectados e seus históricos de tratamento, geraram um procedimento diagnóstico nomeado coprosoroepidemiologia que refletiu mais precisamente a prevalência local. Confirmou-se que a vigilância epidemiológica evitaria o recrudescimento da prevalência. A substituição de Biomphalaria glabrata por Melanoides tuberculata no principal foco de transmissão, contribuiu para a queda acentuada dos índices de infecção.

Esquistossomose Mansoni; Diagnóstico; Doenças Endêmicas

\section{Colaboradores}

M. M. L. Gonçalves e M. S. Soares participaram de todas as etapas do estudo, desde sua concepção e delineamento, coleta, análise e interpretação de dados como redação e revisão crítica do artigo, sendo responsáveis por sua versão final. L. Rey contribuiu no delineamento do estudo, discussão dos resultados e na versão final do artigo. M. G. M. Barreto foi responsável pela organização do banco de dados e análise estatística, também contribuiu na redação, revisão crítica do artigo e na elaboração da versão final. V. R. Maione participou na coleta e construção do banco de dados e contribuiu na análise, interpretação e discussão dos resultados. A. Maldonado Jr. contribuiu na definição e redação da metodologia de diagnóstico e na discussão dos resultados.

\section{Agradecimentos}

Às comunidades de Sumidouro que colaboraram neste trabalho; ao laboratorista Abner Jardim, funcionário da Fundação Nacional de Saúde; aos biólogos Marli Tex, Susana Balmant Simões e Juberlan Garcia pelo auxílio na realização da coproscopia e ao Dr. José Borges Pereira pelo diagnóstico clínico.

\section{Referências}

1. De Vlas SJ, Gryseels B. Underestimation of Schistosoma mansoni prevalences. Parasitol Today 1992; 8:274-9.

2. Rabello ALT. Diagnosing schistosomiasis. Mem Inst Oswaldo Cruz 1997; 92:669-76.

3. Kongs A, Marks G, Verlé P, Van der Stuyft P. The unreliability of Kato-Katz technique limits its usefulness for evaluating $S$. mansoni infections. Trop Med Int Health 2001; 6:163-9.

4. Doenhoff MJ, Chiodini PL, Hamilton JV. Specific and sensitive diagnosis of schistosome infection: can it be done with antibodies? Trends Parasitol 2004; 20:35-9.

5. Doumenge JP, Mott KE, Cheung C, Villenave D, Chapuis O, Perrin MF, et al. Atlas of the global distribution of schistosomiasis. Geneva: World Health Organization; 1987.

6. Fundação Nacional de Saúde, Ministério da Saúde. Controle da esquistossomose: diretrizes técnicas. 1a Ed. Brasília: Fundação Nacional de Saúde, Ministério da Saúde; 1994.

7. D'Andrea PS, Horta C, Cerqueira R, Rey L. Breeding of the water rat (Nectomys squamipes) in the laboratory. Lab Anim 1996; 30:369-76.
8. D'Andrea PS, Gentile R, Cerqueira R, Grelle CEV, Horta C, Rey L. Ecology of small mammals in a Brazilian rural area. Rev Bras Zool 1999; 16:611-20.

9. D'Andrea PS, Maroja LS, Gentile R, Cerqueira R, Maldonado Jr. A, Rey L. The parasitism of Schistosoma mansoni (Digenea-Trematoda) in a naturally infected population of water rats, Nectomys squamipes (Rodentia-Sigmodontidae) in Brazil. Parasitology 2000; 120 (Pt 6):573-82.

10. Minayo MCS. O desafio do conhecimento: pesquisa qualitativa em saúde. 4a Ed. São Paulo: Editora Hucitec/Rio de Janeiro: ABRASCO; 1996.

11. Katz N, Chaves A, Pellegrino J. A simple device for quantitative stool thick-smear technique in schistosomiasis mansoni. Rev Inst Med Trop São Paulo 1972; 14:397-400.

12. Lutz A. O Schistosomum e a schistossomose segundo observações feitas no Brasil. Mem Inst Oswaldo Cruz 1919; 11:121-50.

13. Colley DG, Cook JA, Freeman GL, Bartholomew $\mathrm{RK}$, Jordan P. Immune responses during human schistosomiasis mansoni. I. In vitro lymphocyte blastogenic responses to heterogeneous antigen preparations from schistosome eggs, worms and 
cercariae. Int Arch Allergy Appl Immunol 1977; 53:420-33.

14. Weiland G. The significance of immunodiagnosis in schistosomiasis control: a brief review. Trop Med Parasitol 1989; 40:220-1.

15. Jordan P, Webbe G. Epidemiology. In: Jordan P, Webbe G, editors. Schistosomiasis: epidemiology, treatment and control. London: William Heinemann Medical Books; 1982. p. 87-158.

16. World Health Organization. The control of schistosomiasis: second report of the WHO Expert Committee. Geneva: World Health Organization; 1993. (WHO Technical Report Series 830).

17. Pointier JP, Jourdane J. Biological control of the snail hosts of schistosomiasis in areas of low transmission: the exemple of the Caribbean area. Acta Trop 2000; 77:53-60.

18. Pointier JP. Invading freshwater snails and biological control in Martinique Island, French West Indies. Mem Inst Oswaldo Cruz 2001; 96 Suppl:6774 .
19. Guimarães CT, Souza CP, Soares D. Possible competitive displacement of planorbids by Melanoides tuberculata in Minas Gerais, Brazil. Mem Inst Oswaldo Cruz 2001; 96 Suppl:173-6.

20. Giovanelli A, Vieira MV, Silva CLPAC. Interaction between the intermediate host of schistosomiasis in Brazil Biomphalaria glabrata (Planorbidae) and a possible competitor Melanoides tuberculata (Thiaridae): I. Laboratory experiments. Mem Inst Oswaldo Cruz 2002; 97:363-9.

21. Soares MS, Roque OC, Barbosa CS, Silva LFS, Baptista DF, Valadares J, et al. Enfrentamento de doenças transmissíveis de origem sócio-ambiental: prevenção de impasses pelo diálogo entre disciplinas, setores e sujeitos. Série Fiocruz: Eventos Científicos 2. In: I Seminário de Saúde e Ambiente no Processo de Desenvolvimento. Rio de Janeiro: Fundação Oswaldo Cruz; 2000. p. 101-10.

Recebido em 09/Mar/2004

Versão final reapresentada em 20/Jul/2004 Aprovado em 02/Ago/2004 